\title{
A study of effect of gestational diabetes on the newborn
}

\author{
Rajith M.L ${ }^{1}$, Punyashree $\mathbf{R}^{2}$ \\ ${ }^{1}$ Dr. Rajith M.L, Assistant Professor, Department of Pediatrics, ${ }^{2}$ Dr. Punyashree R, Assistant Professor, Department of \\ Obstetrics and Gynaecology, both authors are affiliated with Sri Siddhartha Medical College, Agalakote, Tumkur, \\ Karnataka, India.
}

Address for Correspondence: Dr. Rajith M.L, Department of Pediatrics, Sri Siddhartha Medical College, Agalakote, Tumkur, Karnataka (State), India. Email:drrajithml@gmail.com

\begin{abstract}
Introduction: Gestational diabetes mellitus has become the nightmare of the modern mothers due to its varied ill effects on the baby. As many of the ill effects can be well taken care with proper education, anticipation and expertise of newborn care taker, this study will throw light on the incidence of those ill effects. Material \& methods: It's a prospective case control study done over a span of one year in a rural medical college in Karnataka. Result: Large for gestational age, Congenital anomalies, Respiratory distress syndrome, Polycythemia, Hypoglycemia were the complications significantly associated with gestational diabetes mellitus. Conclusion: Even our study emphasised the ill effects of gestational diabetes on the baby, RDS, Large baby, Metabolic disorders, Congenital anomalies, polycythemia were significantly associated with gestational diabetes. Most of the complications of GDM on babies are manageable and outcome can be drastically improved. Early diagnosis, education and anticipation of the complications and prompt referral are the need of the hour in peripheries and advancement of neonatal intensive cares in the referral hospitals is needed.
\end{abstract}

Keywords: Polycythemia, Hypoglycemia, Birth asphyxia, Gestational diabetes mellitus

\section{Introduction}

With the improvement of economy of the people, life style diseases like diabetes have jeopardised the quality of life of the people. Gestational diabetes mellitus (GDM) in particular has become a nightmare for women of childbearing age. The outcome and the effect on the child of the GDM pregnancy have the utmost concern due to the varied complications on baby.

Infants born to mothers with glucose intolerance are at an increased risk of morbidity and mortality related to the following: Respiratory distress, Growth abnormalities (large for gestational age (LGA), small for gestational age (SGA), Hyper viscosity secondary to polycythemia, Hypoglycemia, Congenital malformations, Hypocalcemia, hypomagnesemia, and iron abnormalities.

Manuscript received: $20^{\text {th }}$ April 2017

Reviewed: $30^{\text {th }}$ April May 2017

Author Corrected: $7^{\text {th }}$ May 2017

Accepted for Publication: $15^{\text {th }}$ May 2017
These infants are likely to be born by caesarean delivery for many reasons, among which are such complications as shoulder dystocia with potential brachial plexus injury related to the infant's large size.

These mothers must be closely monitored throughout pregnancy. If optimal care is provided, the perinatal mortality rate, excluding congenital malformations, is nearly equivalent to that observed in normal pregnancies.

Communication between members of the perinatal team is of crucial importance to identify infants who are at the highest risk for complications from maternal diabetes. Fetal congenital malformations are most common when maternal glucose control has been poor during the first trimester of pregnancy.

As such, the need for preconceptional glycemic control in women with diabetes cannot be overstated. Maternal 
hyperglycemia during late gestation is more likely to lead to fetal macrosomia, hypoxia, polycythemia, and cardiomegaly with outflow tract obstruction. [1,2,3].

Fetal macrosomia: occurs in $15-45 \%$ of diabetic pregnancies. It is most commonly observed as a consequence of maternal hyperglycemia. $[4,5,6]$.

Impaired fetal growth: may occur in as many as $20 \%$ of diabetic pregnancies, compared with a $10 \%$ incidence for infants born to mothers without diabetes. Maternal renovascular disease is the common cause of impaired fetal growth in pregnancies complicated by maternal diabetes.

Perinatal asphyxia: more common in infants with impaired fetal growth.

Pulmonary disease: These infants are at an increased risk of respiratory distress syndrome and may present within the first few hours after birth. Operative delivery due to macrosomia also increases the risk for transient tachypnea of the newborn, whereas polycythemia predisposes the infant to persistent pulmonary hypertension of the newborn. Hypoglycemia The overall risk of hypoglycemia is anywhere from $25-40 \%$, with LGA and preterm infants at highest risk.

Hypocalcemia or hypomagnesemia: Sixty-five percent of all infants of diabetic mothers (IDMs) demonstrate abnormalities of iron metabolism at birth.

Hematologic problems: Hyperviscosity due to polycythemia increases the IDM's risk for stroke, seizure, necrotizing enterocolitis, and renal vein thrombosis.

Thrombocytopenia: Thrombopoiesis may be inhibited because of an excess of RBC precursors within the bone marrow as a result of chronic in utero hypoxia and increased erythropoietin concentration.
Hyperbilirubinemia: This is common, especially in association with polycythaemia.

Cardiovascular anomalies: Cardiomyopathy with ventricular hypertrophy and outflow tract obstruction may occur in as many as $30 \%$ of IDMs. [7]. These infants are also at an increased risk of congenital heart defects, including ventricular septal defect (VSD) and transposition of the great arteries (TGA).

Congenital malformations: Central nervous system (CNS) malformations are 16 times more likely in IDMs. In particular, the risk of anencephaly is 13 times higher, whereas the risk of spina bifida is 20 times higher. The risk of caudal dysplasia is up to 600 times higher in these infants [8]. Neurologic immaturity, demonstrated by immature sucking patterns, has been found in infants born to insulin-managed mothers with diabetes [9].

Studies in fetal sheep indicate that this may be a reflection of the abnormal brain metabolism and electroencephalogram (EEG) findings as a result of the fetal hyperglycaemia [10]. Renal (hydro nephrosis, renal agenesis, ureteral duplication), ear, gastrointestinal (duodenal or anorectal atresia, small left colon syndrome), and, as mentioned earlier, cardiovascular (single umbilical artery, ventricular septal defect, atrial septal defects, TGA, coarctation of the aorta, cardiomegaly) anomalies are more frequent in these infants.

In developing countries like India these are more stressing due to unavailability of good neonatal care which a infant of diabetic mother badly needs. Studies showing the outcome of infant born to gestational diabetes in developing countries are deficient for planning of care and referral needs. This study was conducted in the medical college in one of the remote district of Karnataka which caters the majority rural population. So, this study will represent the rural population which is most needed.

\section{Material and methods}

Study design: Prospective case control study.

Setting: Rural medical college of Karnataka, India.

Inclusion: Pregnant ladies coming to outpatient department of Obstetrics \& gynaecology department.

Exclusion criteria: Pregnant ladies with overt diabetes and with other comorbid medical conditions, were not included in the study.

Participants: All pregnant women coming for antenatal check-up and willing to follow up at our centre 
Study size: 1000

Period: one year from June 2010 to June 2011.

Over the study period one thousand pregnant women coming to obstetrics and gynaecology department were selected. Seventy five pregnant women proven of gestational diabetes according to WHO criteria were included in study.

Pregnant ladies with other associated comorbidities were not included in the study. They were followed up till the delivery. Perinatal events and complications of all those pregnancies were documented.

\section{Observations}

Table No-1: Gestational age of the newborns.

\begin{tabular}{|c|c|c|}
\hline & Non-GDM (n=925) & GDM (n=75) \\
\hline <30 weeks & 42 & 15 \\
\hline 30-37 weeks & 45 & 47 \\
\hline 37-40 weeks & 750 & 7 \\
\hline >40weeks & 88 & \\
\hline
\end{tabular}

Among babies born to GDM mothers 8\% (6) were less than 30 weeks, 20\%(15) were between 30-37 weeks \& 72\%(47) were between 37-40 weeks.

Table No 2: Sex of the newborns.

\begin{tabular}{|c|c|c|}
\hline Sex & Non-GDM (n=925) & GDM (n=75) \\
\hline Male & 500 & 30 \\
\hline Female & 425 & 35 \\
\hline
\end{tabular}

Among babies born to GDM mothers 53\% (40) \& 47\% (35) were male \& female respectively.

Table No-3: Type of delivery.

\begin{tabular}{|c|c|c|}
\hline & Non-GDM (n=925) & GDM (n=75) \\
\hline Normal vaginal delivery & 550 & 30 \\
\hline Caesarean section & 375 & 35 \\
\hline
\end{tabular}

$53 \%(40) \& 47 \%(35)$ of deliveries were Normal and LSCS respectively in GDM mothers.

Table No-4: Outcome of GDM and Non GDM pregnancies.

\begin{tabular}{|c|c|c|c|c|}
\hline & Outcome & $\begin{array}{c}\text { Non-GDM } \\
(\mathbf{n = 9 2 5})\end{array}$ & $\begin{array}{c}\text { GDM value } \\
(\mathbf{n = 7 5})\end{array}$ \\
\hline 1 & Normal & $786(85)$ & $24(32)$ & $<0.001$ \\
\hline 2 & IUD & $28(3)$ & $10(13.33)$ & $>0.05$ \\
\hline 3 & Still birth & $30(3.5)$ & $3(4)$ & $>0.05$ \\
\hline
\end{tabular}


Original Research Article

\begin{tabular}{|c|c|c|c|c|}
\hline 4 & IUGR & $30(3.5)$ & $3(4)$ & $>0.05$ \\
\hline 5 & LGA & $14(1.5)$ & $21(28)$ & $<0.001$ \\
\hline 6 & Congenital anomalies & $14(1.5)$ & $3(2.5)$ & $<0.05$ \\
\hline 7 & RDS & $37(4)$ & $11(14.67)$ & $<0.001$ \\
\hline 8 & Other Metabolic abnormalities & $37(4)$ & $5(6.67)$ & $<0.001$ \\
\hline 9 & Polycythemia & $24(4)$ & $7(9.33)$ & $<0.001$ \\
\hline 10 & Hypoglycemia & $24(4)$ & $27(36)$ & $<0.001$ \\
\hline
\end{tabular}

Among babies born to GDM mothers, 32\% were Normal, $13 \%$ were IUD, $4 \%$ were Still birth, $4 \%$ were IUGR, $28 \%$ were LGA, $2.5 \%$ were having Congenital anomalies, $14.67 \%$ had RDS, $6.67 \%$ were having Other Metabolic abnormalities.

\section{Discussion}

Macrosomia: The infants of diabetic mothers are traditionally portrayed as being big. The birth weight pattern in our study differed remarkably between gestational diabetics and normal pregnant women. The average birth weights (mean + SD) were $2923+878$. In GDM, maximum babies showed weights more than $3 \mathrm{~kg}$. Babies with birth weight $>4000 \mathrm{gm}$ are called macrosomic. We have noticed $28 \%$ incidence of macrosomia in GDM and was significantly associated. Smiti Nanda et al [11] have reported the mean birth weight of the babies born to gestational diabetic mothers of $3075+326 \mathrm{gm}$ versus the babies born to mothers without GDM of $2790+473$ gm. The difference between mean birth weights in the two groups came out to be statistically significant. Witznitzer et al [12] also observed similar results.

Fetal Distress Syndrome: In our study the overall incidence of fetal distress syndrome in gestational diabetic group was $14.67 \%$ and was significantly associated. It was more common in multigravida than primigravida. All of these patients had hyperglycemia and hyperinsulinemia. Shelly Macfarlane and Tsakalakos et al[13] have observed increased plasma lactate level and decreased $\mathrm{pH}$ in presence of hyperglycemia and hyperinsulinemia. According to them hyperinsulinemia was associated with an increased demand for oxygen leading to fetal hypoxia when the demand could not be fully met as for example in labour. Like Macfarlane and Tsakalakos, we attribute the increased rate of FDS in GDM to hyperglycemia and hyperinsulinemia leading to fetal hypoxia.

Congenital Anomalies: In the present study, nearly $2.5 \%$ cases of GDM delivered babies with obvious congenital abnormality and were significantly associated. Cousins have reported that 2-3\% of newborns had a congenital abnormality at birth in normal pregnancy and the incidence ranged between $7.5 \%$ and $12.9 \%$ in diabetic pregnancies. In view of Ralf Tamura [14], it was estimated to occur with a frequency of $3 \%$ to $6 \%$ in diabetic women which was two to three fold greater than that of nondiabetic population. Our findings are nearer to those of Cousins Many factors have been claimed in pathogenesis of congenital anomalies in pregnancy (like hyperglycemia, ketoacidosis, deficiency of arachadonic acid and myoinositol, excess of sorbitol etc.) by many authors.

According to Miller et al [15] the anomalies occur by the 9th week of amenorrhoea when organogenesis is maximum. Maternal hyperglycemia at this particular period is associated with major structural defects. Pinter et al [16] studied the mechanism by which hyperglycemia produced dysmorphogenesis and proposed it to be hyperglycemia induced yolk sac failure and resulting compromise of nutrient transfer and oxygenation to the early embryo. Hod et al [17] reported a hyperglycemia-associated increase in sorbitol and an inversely proportional reduction in 12 myoinositol levels. Goldman and associates [18] suggested that hyperglycemia induced embryopathy was mediated by afunctional deficiency of arachidonic acid. Data from a study conducted by Mills did not totally corroborate these findings. The Expert Committee on the Diagnosis and Classification of Diabetes Mellitus also did not find any association between a presence of GDM and congenital anomalies [19]. However our results are matching with Miller et al and Goldman et al [15]. 
Looking back that organogenesis occurs rapidly in the first trimester and this is a vulnerable period for congenital anomalies and difficulty to differentiate between true GDM and overt diabetes in first few weeks of gestation, at the most we can conclude that hyperglycemia in first few weeks of gestation (may be of any origin) is probably associated with occurrence of congenital defects.

Still Birth: Incidence of still birth was $4 \%$ in our study and was not significantly associated. Previously many investigators found a strong association between stillbirth and GDM. But today in the modern era of Obstetrics and with improvement in the therapeutic approaches, this complication is no more strongly associated with GDM. However as reported by Hollingsworth, they still occur in women with no prenatal care or unrecognized and /or untreated gestational diabetes who present in at $>41$ weeks of gestation with no ${ }^{20}$ fetal movement. Mansha Dubey and Das reported still birth $[21,22]$ to be an important complication of GDM. Probable explanation for still birth is provided by Salvesen and colleagues who reported decreased fetal $\mathrm{pH}$, and increased $\mathrm{pCo} 2$, lactate and erythropoietin suggestive of fetal acidosis in gestational diabetic women. They further added that hyperglycemia-mediated chronic aberrations in transport of oxygen and fetal metabolites might have produced still births [23]. Kristina Adams did not show a single case of still birth in unrecognized GDM and non-diabetic control [24] groups. The incidence of still birth was found to be only $2 \%$ in GDM patients and still low in non-diabetic pregnant [25] women by Andres Aberg et al. Our results are also in accordance with those of Kristina Adams and Andres Aberg. Hyperglycemia per say alone is not a threatening factor for stillbirth unless and until other unfavourable factors operate simultaneously. Nowadays due to appropriate care and monitoring during antenatal period, occurrence of stillbirth is not increased in gestational diabetes mellitus.

IUD, IUGR: In our study, we noticed that incidence rate of IUD was $13 \%$ and IUGR was $4 \%$. And there was no significant difference between babies of diabetic mother and another normal group. Many investigators have reported an increased incidence rate of IUD and IUGR in the past but nowadays it is very low. Mondestein et al found that lower birth weight babies, smaller babies and growth-restricted babies had an increased risk of fetal death.

Hypoglycemia: Incidence in our study was $36 \%$ and was significantly associated. Polycythemia: Incidence was $9.33 \%$ and was significantly associated.

\section{Conclusion}

Even our study emphasised the ill effects of gestational diabetes on the baby, RDS, Large baby, Metabolic disorders, Congenital anamolies polycythemia were significantly associated with gestational diabetes. And one good thing is most of the complications of GDM on babies are manageable and outcome can be drastically improved. Early diagnosis, education and anticipation of the complications and prompt referral is the need of the hour in peripheries and advancement of neonatal intensive cares in the referral hospitals is needed.

\section{Abbreviations:}

GDM: Gestational diabetes mellitus

IDM: Infant of diabetic mother

VSD: Ventricular septal defect

ASD: Atrial septal defect

TGA: Transposition of great arteries

LGA: Large for gestational age

SGA: Small for gestational age

EEG: Electro encephalogram

Funding: Nil, Conflict of interest: None initiated, Perission from IRB: Yes

\section{References}

1. Barnes-Powell LL. Infants of diabetic mothers: the effects of hyperglycemia on the fetus and neonate. Neonatal Netw. 2007 Sep-Oct. 26(5):283-90.DOI: DOI: 10.1891/0730-0832.26.5.283

2. Plagemann A. A matter of insulin: developmental programming of body weight regulation. J Matern Fetal Neonatal Med. 2008 Mar. 21(3):143-8. DOI: 10. 1080/14767050801929869.

3. Boney CM, Verma A, Tucker R, Vohr BR. Metabolic syndrome in childhood: association with birth weight, maternal obesity, and gestational diabetes mellitus. 
Pediatrics. 2005 Mar. 115(3):290-6. DOI: 10.1542/ peds.2004-1808

4. Mäkelä J, Lagström $\mathrm{H}$, Kaljonen A, Simell O, Niinikoski H. Hyperglycemia and lower diet quality in pregnant overweight women and increased infant size at birth and at 13months of age - STEPS study. Early Hum Dev. 2013 Jun. 89(6):439-44. DOI: 10.1016/j. earlhumdev. 2013.01.007

5. Plagemann A, Harder T, Rodekamp E, Kohlhoff R. Rapid neonatal weight gain increases risk of childhood overweight in offspring of diabetic mothers. J Perinat Med. 2012 Sep. 40(5):557-63. DOI: 10.1515/jpm-20110270 .

6. Maayan-Metzger A, Schushan-Eisen I, Strauss T, Globus O, Leibovitch L. Gestational weight gain and body mass indexes have an impact on the outcomes of diabetic mothers and infants. Acta Paediatr. 2015 Nov. 104 (11): 1150-5. doi: 10. 1111/apa. 13166.

7. Elmekkawi SF, Mansour GM, Elsafty MS, Hassanin AS, Laban M, Elsayed HM. Prediction of Fetal Hypertrophic Cardiomyopathy in Diabetic Pregnancies Compared with Postnatal Outcome. Clin Med Insights Womens Health. 2015. 8:39-43. doi: 10.7860/ JCDR/ 2016/17993.8079

8. DeBoer T, Wewerka S, Bauer PJ, Georgieff MK, Nelson CA. Explicit memory performance in infants of diabetic mothers at 1 year of age. Dev Med Child Neurol. 2005 Aug;47(8):525-31.

9. Bromiker R, Rachamim A, Hammerman C, Schimmel M, Kaplan M, Medoff-Cooper B. Immature sucking patterns in infants of mothers with diabetes. J Pediatr. 2006 Nov;149(5):640-3.

10. Rosenkrantz TS, Knox I, Zalneraitis EL, et al. Cerebral metabolism and electrocortical activity in the chronically hyperglycemic fetal lamb. Am J Physiol. 1993 Dec. 265(6 Pt 2):R1262-9. PMID: 8285266

11. Smiti Nanda, Umber Agarwal, Harbans Lal, et al. Absence of fasting hyperglycemia in Gestational Diabetics as a predictor of good perinatal outcome in high risk population- A study from northern India. Indian Medical Gazette, March (2002), 75-77.
12. A.Wiznitzer, D.Fraser, S.Weitzman et al. A prospective clinical trial of gestational diabetes vs. controls: Perinatal Outcomes. Am J Obstet Gynecol, 178(1): 53-60.

13. MacFarlane C M, Tsakalakos N. Relative fetal hypoxia as a contributing factor to fetal macrosomia in diabetic pregnancy. Medical Hypothesis, 1983, 11: 365-374.

14. Tamura R K, Sabbagha R E, Depp R et al: Diabetic macrosomia: Accuracy of third trimester ultrasound. Obstet Gynecol, 1986, 67:828-834.

15. Mills J L: Malformations in infants of diabetic mothers. Teratology, 1982, 25: 385. DOI: 10.1002/ bdra. 20757

16. Pinter E, Reece E A, Leranth C Z, et al. Yolk sac failure in embryopathy due to hyperglycaemia. Teratology, 1986, 33 (1): 73-84. DOI: 10.1002/tera. 1420330110

17. Hod M, Star S, Passonnequ J V, et al. Effect of hyperglycemia on sorbitol and myoinositol content of cultured rat conceptus : Failure of aldose reductase inhibitors to modify myoinositol depletion and dysmorphogenesis. Biochem Biophys Res Commun, 1986, 140: 974.

18. Goldman A S, Baker L, Piddington $\mathrm{R}$, et al Hyperglycaemia induced teratogenesis is mediated by a functional deficiency of arachidonic acid. Proceedings of the National Academy of Sciences of the USA, 1985, 82 (23): 8227-8231.

19. Report of the Expert Committee on the Diagnosis and Classification of Diabetes Mellitus. Diabetes Care. 1997 Jul;20(7):1183-97.

20. Hollingsworth DR, Grundy S M. Pregnancy associated hypertriglyceridemia in normal and diabetic women: Differences in insulin dependent, non-insulin dependent and gestational diabetes. Diabetes, 1982, 31: 1092-1097.

21. Mansha Dubey. Diabetes in Pregnancy. JAMA India- The Physicians' Update 4(12): 66-68. 
22. Das UN. Gestational diabetes mellitus. J Assoc Physicians India. 2001 Mar;49:361-5.

23. Salvesen DR, Brudenell MJ, Nicolaides KH. Fetal polycythemia and thrombocytopenia in pregnancies complicated by maternal diabetes mellitus. Am J Obstet Gynecol. 1992 Apr;166(4):1287-93.
24. Kristina M. Adams, Hongzhe Li, Roger L. Nelson et al. Sequelae of unrecognized gestational diabetes. Am J Obstet Gynecol, 1998, 178(6): 1321-1331.

25. Aberg A, Rydhstroem H, Frid A. Impaired glucose tolerance associated with adverse pregnancy outcome: a population-based study in southern Sweden. Am J Obstet Gynecol. 2001 Jan;184(2):77-83.

\section{How to cite this article?}

Rajith M.L, Punyashree R. A study of effect of gestational diabetes on the newborn. J PediatrRes.2017;4(05):303309.doi:10. 17511/ijpr.2017.i05.03 